\title{
Strategy from the Perspective of Supply Chain
}

\author{
${ }^{1}$ Shandong University, Jinan, China \\ ${ }^{2}$ Shanxi Cambridge International School, Jinzhong, China \\ ${ }^{3}$ Stevenson International School, Shanghai, China \\ ${ }^{4}$ Frederick Gunn School, Washington, USA \\ *Correspondence author email: 2373554769@qq.com \\ + They are all the first authors.
}

Fanchen Meng ${ }^{1,+,}$, Yanqiao Jin ${ }^{2,+}$, Xinyu $\mathrm{Miao}^{3,+}$, Ruhua $\mathrm{Pan}^{4,+}$

\begin{abstract}
Promotion is more common in the fashion product sales. In this paper, the authors built a customer supply chain model of both myopic and strategic consumers with the two period of sales, and then seek to deduce a centralized decisionmaking and decentralized decision-making model consisting of two kinds of pricing decisions. The result shows that the supply chain revenue under the decentralized decision-making model is less than the supply chain revenue under the centralized decision-making model. Meanwhile, in Collective decision model, the manufacturer's revenue is greater than the revenue of each retailer, and it is also greater than the sum of the retailer's revenue.
\end{abstract}

Keywords: Supply Chain, customer behavior, two-period pricing, marketing

\section{INTRODUCTION}

Two-stage (normal sales and discounts) often appear in the commodity sales activities, where goods are sold on the normal sales period at a certain price, and on final sales or clearance stage sales at a relatively low price, this phenomenon is more common in the fashion product sales, such as fashion clothes, fashion bags, and electronic products, etc. At the same time, some consumers are getting smarter about the exact time to purchase the product. Retailers express great disgust at such consumption behavior of customers, calling customers who wait for price cuts, promotions and discounts "demons" and those who buy decisively "angels". Wal-Mart, the giant retailer, gets $20 \%$ of its annual sales during its Christmas and Thanksgiving sales. Clearly, some customers are becoming more strategic, deciding whether to buy now or wait for the discount period based on their utility for the product in different time periods. Customers who express a delay in their purchase for price cuts are defined as strategic customers, while customers with no delayed purchase behavior are defined as myopic customers.

Scholars have done a lot of research on customer behavior and two-period pricing. Zhang and Cooper [1] analyzed the influence that the strategic customer behavior would pose on the set price and limited quantity decision for firms with two-period pricing. Yang Hui et al. analyzed the dynamic pricing of consumers' myopic and strategic behaviors. Kremer et al. [2] analyzed the dynamic pricing problem of retailers facing myopic and strategic customers. Su and Zhang [3] analyzed the impact of customer strategic behavior on supply chain performance under wholesale price contracts. Ji Guojun et al. analyzed the influence of strategic customer behavior on optimal decision-making and performance of supply chain member enterprises under three different power structures, which they found that if supply chain member enterprises face up to the existence of strategic customers, they can achieve a mutually beneficial situation by making reasonable decisions. Recently, Zhang et al. [4] proposed a two-phase pricing model for a supply chain consisting of a single manufacturer and a single retailer and discussed the influence of reference price on supply chain decision-making. Xue et al.[5] analyzed with the presence of revenue sharing contracts to coordinate the secondary supply chain where customers had "show-off" behavior.

In the paper, we set up a customer supply chain model of both myopic and strategic consumers with the two period of sales, and then seek to deduce a centralized decision-making and decentralized decision-making model consisting two kinds of pricing decisions. 


\section{MODELLING}

\subsection{Model Description}

Consider a two-stage supply chain consisting of one manufacturer and $\boldsymbol{n}$ homogeneous retailers. The retailers order a single product produced by the manufacturer and sells it to the customer at different prices in two periods. Since the products sold by retailers are of the same quality, in the same market, the final prices of the products they sell must be the same. The sales price in the regular sales period is $p_{1}$, and the sales price in the discount period is $p_{2}\left(0<p_{2}<p_{1}\right)$. The manufacturer's production cost per unit product is referred as $c$, the manufacturer would decide the wholesale price of the product $\boldsymbol{w}$; and the retailers would decide the sales price $p_{1}$ and $p_{2}$.

Assume that at the beginning of the selling season the retailer announces its price path and keeps its promise. It is assumed that the size of the market is certain, and in order to lose generality, it is normalized to 1 . The maximum willingness to pay (MWP) of each customer for the product is $\mathrm{V}$. It is assumed that $\mathrm{V}$ is uniformly distributed on $[0,1]$. All customers arrive at the market at the beginning of the sales period and stay there until the product is purchased or the sales period ends.

There are two types of customers in the market: myopic customers and strategic customers. Suppose that the proportion of myopic customers and strategic customers in the market is represented as $\alpha$ and $1-\alpha$, respectively, where $0<\alpha<1$. Myopic customers decide whether to buy or not according to their instant utility and they will only buy in full-price period; The strategic customer compares the utility of the purchase between the two sales stages and then determines the purchase period. That is to say, as long as the myopic customer's maximum willingness to pay is greater than or equal to the current selling price, the purchase behavior will occur immediately. $\theta$ is the utility discount factor of strategic customers in the discount period, and $0<\theta<$ 1 , that is to say, the utility of strategic customers will decrease when they delay their purchase to the discount period.

Assuming that the utility discount factor of the purchase of the strategic customer in the discount period is the same, $\theta$ can also be understood as "degree of strategic behavior" or "tolerance". According to the hypothesis, when strategic customers purchase in the first stage, their willingness to pay $v$ meets: $v-p_{1}=$ $\left(v-p_{2}\right) \theta, \quad v-p_{1}>0$

Here, the critical value $\hat{v}=\frac{p_{1}-\theta p_{2}}{1-\theta}$ can be obtained. Thus, the number of strategic customers who purchase in the normal sales period is $(1-\alpha)\left(1-\frac{p_{1}-\theta p_{2}}{1-\theta}\right)$, and the number of strategic customers who purchase in the discount period is $(1-\alpha)\left(\frac{p_{1}-\theta p_{2}}{1-\theta}-p_{2}\right)$, so the total number of customers who purchase in the normal sales period is given by

$$
d_{1}=\alpha\left(1-p_{1}\right)+(1-\alpha)\left(1-\frac{p_{1}-\theta p_{2}}{1-\theta}\right)
$$

whereas since myopic customers only purchase in the normal sales period, so the total number of customers who purchased during the discount period is given by

$$
d_{2}=(1-\alpha)\left(\frac{p_{1}-\theta p_{2}}{1-\theta}-p_{2}\right)(2)
$$

\subsection{Collective Decision model}

In a collective decision model, manufacturers and retailers are regarded as an intact supply chain, and would set the corresponding price in order to maximize the overall profit of the chain.

Under these assumptions, the profit of the overall supply chain can be written as:

$$
\pi_{i}=\left(p_{1}-c\right) d_{1}+\left(p_{2}-c\right) d_{2}
$$

Where $\mathrm{d} 1$ and $\mathrm{d} 2$ were given by equations (1) and (2).

\section{(1) Solution:}

In a collective decision model, the profit-maximizing price in the normal and discount selling period are:

$$
\begin{gathered}
p_{1}=\frac{c(\theta+1)(\alpha-1)}{(\theta+1)^{2}(1-\alpha)+4(\alpha \theta-1)} \\
p_{2}=\frac{(1-\alpha)(\theta-1)(\alpha c-\alpha \theta c+2 c)}{(\theta+1)^{2}(1-\alpha)^{2}-4(\alpha-1)(\alpha \theta-1)}
\end{gathered}
$$

Respectively. The profit in the overall supply chain can be determined by substituting equation (4) and (5) back to (3).

\section{(2) Proof:}

Consider the following Hessian Matrix:

$$
\begin{gathered}
H=\left[\begin{array}{cc}
\frac{\partial^{2} \pi_{i}}{\partial p_{1}^{2}} & \frac{\partial^{2} \pi_{i}}{\partial p_{1} \partial p_{2}} \\
\frac{\partial^{2} \pi_{i}}{\partial p_{2} \partial p_{1}} & \frac{\partial^{2} \pi_{i}}{\partial p_{2}^{2}}
\end{array}\right]= \\
{\left[\begin{array}{cc}
\frac{2(\theta \alpha-1)}{1-\theta} & \frac{(\theta+1)(1-\alpha)}{1-\theta} \\
\frac{(\theta+1)(1-\alpha)}{1-\theta} & \frac{2(\alpha \theta+\alpha-1)}{1-\theta}
\end{array}\right]}
\end{gathered}
$$

In which $0<\alpha<1,0<\theta<1$,

$$
\begin{gathered}
\frac{\partial^{2} \pi_{i}}{\partial p_{1}^{2}}=\frac{2(\theta \alpha-1)}{1-\theta}<0 \\
\frac{\partial^{2} \pi_{i}}{\partial p_{1}^{2}} * \frac{\partial^{2} \pi_{i}}{\partial p_{2}^{2}}-\frac{\partial^{2} \pi_{i}}{\partial p_{2} \partial p_{1}} * \frac{\partial^{2} \pi_{i}}{\partial p_{1} \partial p_{2}}=\left[\frac{2(\theta \alpha-1)}{1-\theta} *\right. \\
\left.\frac{2(\alpha \theta+\alpha-1)}{1-\theta}\right]-\left[\frac{(\theta+1)(1-\alpha)}{1-\theta}\right]^{2}>0 \quad(8)
\end{gathered}
$$

Thus, $\pi_{r i}$ is a joint convex function of $p_{1}$ and $p_{2}$, whose maximum is achieved when $\frac{\partial \pi_{r i}}{\partial p_{1}}=0, \frac{\partial \pi_{r i}}{\partial p_{2}}=0$.

Figure 1 shows a 3-dimensional graph of $\pi_{r i}$ in respect to $p_{1}$ and $p_{2}$ as its variables, which also clearly 
illustrates that the objective function has an absolute maximum.

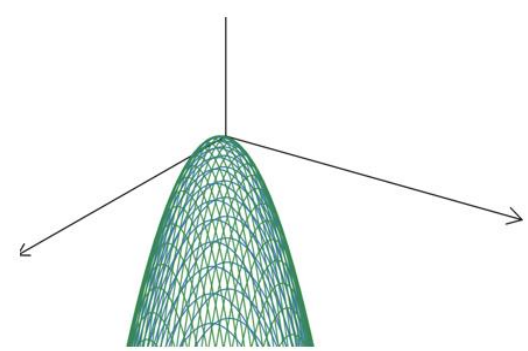

Figure 1 a 3-dimensional

We then solve the equation set given by $\frac{\partial \pi_{r i}}{\partial p_{1}}=$ 0 , $\frac{\partial \pi_{r i}}{\partial p_{2}}=0$, which gives us the value of $p_{1}$ and $p_{2}$ in respect to $c, \alpha, \theta \mathrm{c}$, as constants, as expressed in (4) and (5).

\subsection{Collective Decision model}

Under decentralized decision-making, manufacturers and retailers make decisions on wholesale prices and retail prices with the goal of maximizing their respective profits. We consider the stackelberg game process with the manufacturer as the leader and the retailer as the follower, that is, first, the manufacturer decides the wholesale price, and then the retailer decides the retail price in the two periods. Homogeneous retailer's profit under decentralized decision-making can be expressed as:

$\pi_{r i}=\left(p_{1}-w\right) \frac{d_{1}}{n}+\left(p_{2}-w\right) \frac{d_{2}}{n}, \quad i=$

\section{$1,2,3, \ldots, n \quad(9)$}

The reason why the equation looks like above is because retailers are all homogeneous, the final price of each retailer must be equal, and the market shares to be divided are the same.

The manufacturer's profit is:

(1) Proof

$$
\pi_{m}=(w-c)\left(d_{1}+d_{2}\right)
$$

Since $\pi_{r i}$ is about the joint convex function of $p_{1}$ and $p_{2}$, there is a maximum when

$$
\frac{\partial \pi_{r i}}{\partial p_{1}}=0, \frac{\partial \pi_{r i}}{\partial p_{2}}=0
$$

By combining the solution, the retailer's pricing response function is obtained:

$$
\begin{gathered}
p_{1}(w)=\frac{w(1+\alpha+\theta-\alpha \theta)+2}{\alpha+\theta-\alpha \theta+3} \\
p_{2}(w)=\frac{w(2+\alpha+\theta-\alpha \theta)+\theta+1}{\alpha+\theta-\alpha \theta+3}
\end{gathered}
$$

\section{(1) Solution:}

Substitute $\mathrm{p} 1(\mathrm{w})$ and $\mathrm{p} 2(\mathrm{w})$ into the manufacturer's decision function and utilize $\frac{\partial \pi_{m}}{\partial w}=0$, then we can get the best Wholesale prices:

$$
w^{*}=\frac{c+1}{2}
$$

Below is the standard view of $\pi_{r i}{ }^{*}$, the z-axis is the objective function, the $\mathrm{y}$-axis is the $\alpha$, and the $\mathrm{x}$-axis is the $\theta$.

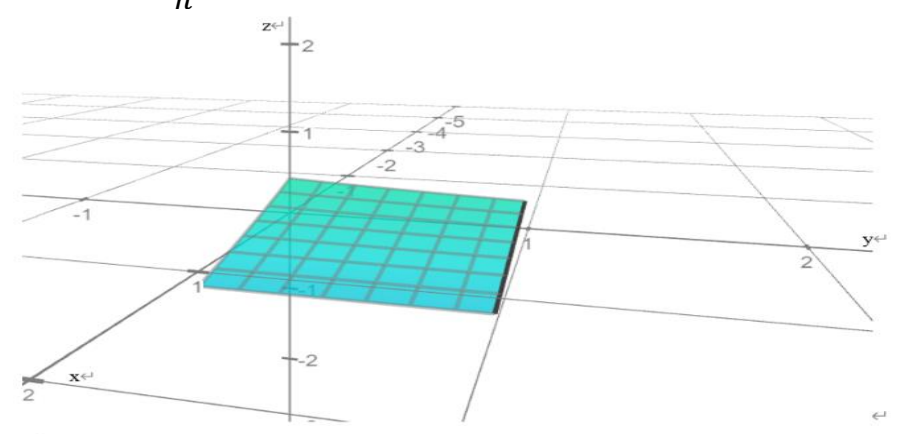

Figure 2 standard view of $\pi_{r i}{ }^{*}$

Below is the standard view of $\pi_{m}{ }^{*}$, the z-axis is the objective function, the $\mathrm{y}$-axis is the $\alpha$, and the $\mathrm{x}$-axis is the $\theta$. 


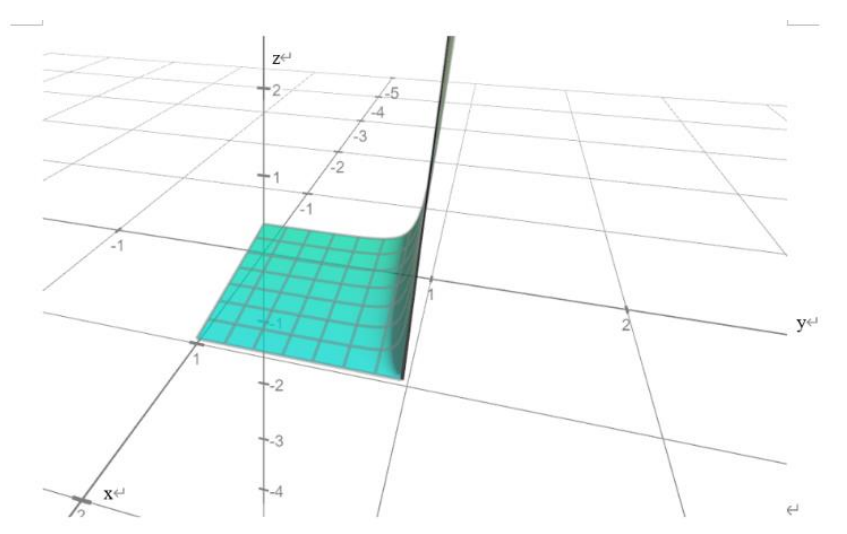

Figure 3 the standard view of $\pi_{m}{ }^{*}$

\section{CONCLUSION}

In a Supply chain under one manufacturer, $n$ homogeneous retailers and multiple customers.

(1) The supply chain revenue under the decentralized decision-making model is less than the supply chain revenue under the centralized decisionmaking model.

(2) In Collective decision model, the manufacturer's revenue is greater than the revenue of each retailer, and it is also greater than the sum of the retailer's revenue. Besides, the manufacture's revenue is indifferent for the change of $\boldsymbol{n}$.

(3) In Decentralized decision model, When $\boldsymbol{n}$ tends to infinity, the retailer's revenue tends to $\boldsymbol{0}$, which means that $\boldsymbol{p} \mathbf{1}$ tends to $\boldsymbol{p} \mathbf{2}$ tends to cost $\boldsymbol{w}$. Besides, the manufacturer's revenue is greater than the revenue of each retailer, and it is also greater than the sum of the retailer's revenue. Investigate the reasons, I think there are two mains: first, Manufacturers are in a monopoly position, while retailers are not. Second, this model is a stackelberg game process assuming that the manufacturer is the leader and the retailer is the follower, so the firstpricing effect has the preemptive effect.

\section{REFERENCES}

[1] Zhang D,Cooper W L. Managing clearance sales in the presence of strategic customer $[\mathrm{J}]$. Production and Opera-tions Management, 2008, 17 (4) :416431.

[2] Kremer K,Mantin B,Ovchinnikov A. Dynamic pricing in the presence of myopic and stratrgic consumers: theory and experiment $[\mathrm{J}]$. Production and Operations Management, 2017, 26 (1): 116-133.

[3] Su X M, Zhang F Q. Strategic customer behavior, commit-ment, and supply chain performance $[\mathrm{J}]$. Management Sci-ence, 2008, 54 (10) :1759-1773.

[4] Zhang J Z,Guo Q L,Zhang J. Supply chain pricing deci-sions with price reduction during the selling season [J]. In-ternational journal of production research,2014, 52(1) : 165-187.

[5] Xue M S, Tang W S, Zhang J X. Supply chain pricing and coordination with markdown strategy in the presence of con-spicuous consumers [J]. International transactions in opera-tional research, 2016, 23 (6) :1-15. 